\title{
Scattering Cancellation-Based Cloaking for the Maxwell-Cattaneo Heat Waves
}

\author{
M. Farhat,,${ }^{1, *}$ S. Guenneau, ${ }^{2}$ P.-Y. Chen, ${ }^{3}$ A. Alù, ${ }^{4}$ and K.N. Salama ${ }^{1}$ \\ ${ }^{1}$ Division of Computer, Electrical, and Mathematical Science and Engineering, \\ King Abdullah University of Science and Technology (KAUST), Thuwal 23955-6900, Saudi Arabia \\ ${ }^{2}$ Aix Marseille Univ, CNRS, Centrale Marseille, Institut Fresnel, Marseille, France \\ ${ }^{3}$ Department of Electrical and Computer Engineering, University of Illinois at Chicago, \\ Chicago, Illinois 60607, USA \\ ${ }^{4}$ Photonics Initiative, Advanced Science Research Center, City University of New York, New York, \\ New York 10031, USA
}

(Received 8 January 2019; revised manuscript received 26 March 2019; published 29 April 2019)

\begin{abstract}
We theoretically propose scattering cancellation-based cloaks for heat waves that obey the MaxwellCattaneo equation. The proposed cloaks possess carefully tailored diffusivity to cancel the dipole scattering from the object that they surround, and thus can render a small object invisible in the near and far fields, as demonstrated by full-wave finite-element simulations. Mantle heat cloaking is further analyzed and proposed to simplify the design and bring this cloaking technology one step closer to its practical implementation, with promising applications in nanoelectronics and defense-related applications.
\end{abstract}

DOI: 10.1103/PhysRevApplied.11.044089

\section{INTRODUCTION}

Ever since the scattering cancellation technique was proposed in 2005 [1], there has been a keen interest in the photonics community and significant studies investigating its intriguing mechanisms and promising applications for different kinds of waves including, but not limited to, microwaves [2], optics [3], elastodynamics [4,5], acoustics [6-8], thermal [9], and matter [10]. Soon afterward, in 2006, Pendry and coauthors proposed first theoretically [11] and then experimentally, to cover a copper disc of 25-mm radius with a cylindrical coating made of split ring resonators, carefully tailored to make the medium heterogeneous in a way to curve the path of light in it. In fact, one says that an object is invisible, if it does not modify (or change by a little) the wave-field in which it is embedded [12]. One speaks of transparency, if the object itself possesses these properties, and of cloaking, if it is surrounded by a device that offers the same functionality. In this way, invisibility cloaks guide waves around the considered area by creating a hole in the space metric. A related, but quite different technique was proposed at the same time by Leonhardt on conformal-mapping-based cloaking [13]. Several other cloaking mechanisms have been put forward in recent years. These range from active-cloaking-based on anomalous localized resonances [14,15], homogenizationbased cloaking [16,17], waveguide theory [18], and the use of invisibility layers that make an object appear to be

*mohamed.d.farhat@gmail.com located outside its domain invisible by employing a complementary "anti-object" integrated inside a shell with a "folded geometry" [19].

When it comes to heat waves, the design of convenient invisibility devices is of paramount importance due to potential applications related to heat exchange, power integration, microelectronics, and even green-building construction or defense [20]. Transformation optics (TO), anomalous resonance (AR), and scattering cancellation techniques (SCT) have been applied toward this goal [2129]. In particular, SCT offers simpler designs than the aforementioned approaches when the size of the objects is smaller than, or comparable to, the wavelength and thus the dipole approximation applies [30]. In this scenario, isotropic layers of specific diffusivity are sufficient to cancel the first-order scattering and thus significantly reduce the scattering cross section (or radar cross section) of the core-shell system. It was even shown that mantle cloaks, that is, ultrathin layers, can mimic invisibility for such kinds of waves [31-34].

In this work, we propose to generalize the concepts of SCT and mantle cloaking to the field of heat waves obeying the Maxwell-Cattaneo equation [35,36]. The Fourier transfer law of heat gives rise to a parabolic equation that results in instantaneous propagation speeds, which is an unphysical feature, as is the case with classical mechanics [37]. This inconsistency is solved in the latter case by the theory of special relativity [38]. Maxwell-Cattaneo law in this sense is a generalization of the Fourier law that results in hyperbolic equations, and therefore, finite speeds 
of propagation. In the following sections, we discuss the details of the cloak design, the physical mechanisms of this scattering cancellation mechanism, and its mantle cloaking realization in the framework of Maxwell-Cattaneo heat waves.

\section{DISPERSION MECHANISM OF MAXWELL-CATTANEO WAVES}

\section{A. Maxwell-Cattaneo equation}

Thanks to all of the aforementioned advantages, we propose to apply the SCT to heat waves using the MaxwellCattaneo formalism. As a matter of fact, when the Fourier law is used to describe the heat transfer, that is,

$$
\Phi=-\kappa_{0} \nabla T
$$

with $\Phi$ the local heat flux density (in $\mathrm{W} \mathrm{m}^{-2}$ ), $\kappa_{0}$ the conductivity of the medium that can be anisotropic (in $\mathrm{W} \mathrm{m}^{-1} \mathrm{~K}^{-1}$ ), and $T$ the temperature field (in $\mathrm{K}$ ), it is well known that this law leads to a parabolic equation for $T$ (the Fourier heat equation). Thus, any initial disturbance in the medium (or object) is propagated instantly due to the parabolic nature of the equation [37]. In order to circumvent this apparent unphysical situation, one can use instead the Maxwell-Cattaneo law, which is one of various widely accepted modifications of the Fourier law [36]. It takes the form

$$
\left(1+\tau_{0} \frac{\partial}{\partial t}\right) \Phi=-\kappa_{0} \nabla T
$$

with $\tau_{0}$ being the thermal relaxation time (that corresponds to the time it takes for the object to reduce its temperature to half. The heat transfer rate thus decays for larger thermal relaxation times) [37,39]. The additional term $\tau_{0} \partial_{t} \Phi$ accounts for thermal inertia, which avoids the so-called phenomenon of infinite propagation. It is also necessary to add another corrective term that accounts for flux diffusion. The Maxwell-Cattaneo equation, as described above, therefore, takes the final form of a system of coupled partial differential equations (PDEs)

$$
\begin{aligned}
& \frac{\partial T}{\partial t}=-\nabla \cdot \Phi, \\
& \tau_{0}\left(\frac{\partial \Phi}{\partial t}-\sigma_{0} \Delta \Phi\right)=-\Phi-\kappa_{0} \nabla \mathrm{T},
\end{aligned}
$$

that can be rewritten as

$$
\tau_{0} \frac{\partial^{2} \mathrm{~T}}{\partial t^{2}}+\frac{\partial T}{\partial t}-\kappa_{0} \Delta T-\tau_{0} \sigma_{0} \Delta\left(\frac{\partial \mathrm{T}}{\partial t}\right)=\delta,
$$

with $\delta$ being the Dirac distribution representing the source term and $\sigma_{0}$ accounting for the diffusive phenomena. This extra diffusive term is a corrective term representing the flux diffusion added to the system of equations in order to make the discretizing process asymptotically stable, when solving the Maxwell-Cattaneo equation by means of the finite difference method for example [40,41].

\section{B. Dispersion relation of the Maxwell-Cattaneo equation}

Assuming a time harmonic incident wave, that is, proportional to $e^{-i \omega t}$, with $\omega$ the angular frequency, Eq. (4) is further simplified to (by omitting the $\delta$ term)

$$
\Delta T+\frac{\omega\left(\omega \tau_{0}+i\right)}{\kappa-i \omega \tau_{0} \sigma_{0}} T=0
$$

By inspection of Eq. (5), one immediately sees that an effective wave number $k_{0}$ can be defined as $k_{0}=$ $\omega \sqrt{\left(\omega \tau_{0}+i\right) /\left(\kappa \omega-i \omega^{2} \tau_{0} \sigma_{0}\right)}$. This means that $k_{0}$ is a complex number for all frequencies, which is markedly different from classical heat waves (Fourier transfer) or even diffusive waves (or diffuse photon density waves, DPDWs) that possess complex wave numbers in specific frequency ranges. This phenomenon is quite understandable, since one expects new physics to be at play [42]. Figure 1(b) plots the dispersion relation, that is, $k_{0}$ vs the normalized frequency $\omega \tau_{0}$, for two specific scenarios. In the upper panel of Fig. 1(b), one assumes an extra term (corresponding to DPDWs) in $k_{0}$, that is, $k_{0}=$ $\omega \sqrt{\left(\omega \tau_{0}+i-1 /\left(\omega \tau_{d}\right)\right) /\left(\kappa \omega-i \omega^{2} \tau_{0} \sigma_{0}\right)}$, with $\tau_{d}$ the lifetime of photons. The second scenario assumes $\tau_{d}=\infty$, or in a sense, the absence of absorption (absence of DPDWs). For the first scenario, we observe three specific spectral domains: in the first one, $\operatorname{Im}\left(k_{0}\right) \gg \operatorname{Re}\left(k_{0}\right)$, where absorption dominates and for $\omega \tau_{0} \rightarrow 0$, there is a convergence toward $k_{0}^{s}=\sqrt{1 /\left(\tau_{d} \kappa_{0}\right)}$ (purely imaginary wave number);

(a)
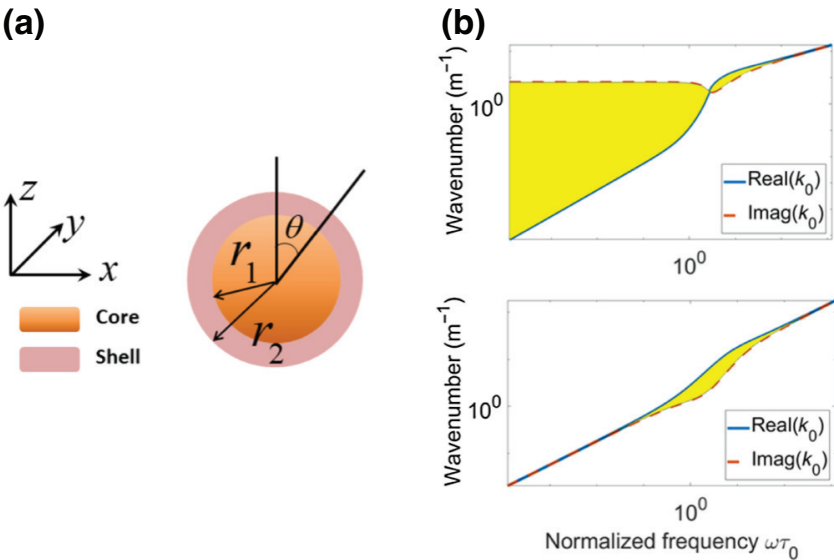

FIG. 1. (a) Schematic representation of the core-shell system in cross-section view in the $x-z$ plane. (b) Dispersion relation for the medium computed using Eq. (5) for the Maxwell-Cattaneo, with (top) and without (bottom) the extra absorption term (corresponding to diffusive waves). 
in the second domain, one has $\operatorname{Re}\left(k_{0}\right) \gg \operatorname{Im}\left(k_{0}\right)$, that is, propagative regime; and in the third domain, one has $\operatorname{Re}\left(k_{0}\right) \approx \operatorname{Im}\left(k_{0}\right)$, that is, the scattering and absorption are balanced. For the second scenario, we have the same behavior for spectral regions 2 and 3, and in region 1, we have $\operatorname{Re}\left(k_{0}\right) \approx \operatorname{Im}\left(k_{0}\right)$, and both converge toward 0 in the static regime since there is no absorption there. In these plots, the parameters are assumed to be $\tau_{0}=0.2 \mathrm{~s}, \sigma_{0}=$ $0.1 \mathrm{~W} \mathrm{~m}^{-1} \mathrm{~K}^{-1}, \kappa_{0}=0.75 \mathrm{~W} \mathrm{~m}^{-1} \mathrm{~K}^{-1}$, and $\tau_{d}=0.03 \mathrm{~s}$.

\section{SCATTERING CANCELLATION FOR THE MAXWELL-CATTANEO EQUATION}

\section{A. Set up of the scattering problem}

We now move to the derivation and analysis of the scattering problem in the framework of Maxwell-Cattaneo heat waves. We assume that incident waves are plane waves (which is a good approximation for spherical waves far away from the source). These plane waves impinge on the core-shell system [spherical object and shell, schematized in Fig. 1(a)] and are shown in the $x-z$ plane. The geometrical and physical parameters of the object and the cloak are identified using subscripts 1 and 2, respectively, while free-space parameters have subscript 0 ( $k_{0}$ as wave number in free space, for example). We further make the assumption that the origin of the spherical coordinates lies in the center of the object without loss of generality. It is thus possible to find solutions to Eq. (5) by developing the temperature field in the different regions of space in terms of spherical Bessel and Hankel functions of different kinds. These eigensolutions are shown to obey the Helmholtz equation with an equivalent (complex) wave number in the argument.

Inside free space, two components of the temperature field (normalized to the incident amplitude, or equivalently assuming a unit-amplitude incident field) exist, namely, the incident field $T^{\text {in }}$, that is, the plane wave $e^{i k_{0} r \cos \theta}$

$$
T^{\text {in }}=\sum_{l=0}^{\infty} i^{l}(2 l+1) j_{l}\left(k_{0} r\right) P_{l}(\cos \theta),
$$

with $P_{l}$ the Legendre polynomial of order $l, j_{l}$ denoting the spherical Bessel function of order $l$, and $\theta$ the incidence angle of the waves, where we took advantage of the spherical symmetry of the problem, and placed the source in the $z$ axis. The second field in free space, and the most important one regarding our problem, is the scattered temperature field $T^{\mathrm{sc}}$, resulting from the inhomogeneity encountered by the wave where it impinges from one medium to another, that is,

$$
T^{\mathrm{sc}}=\sum_{l=0}^{\infty} i^{l}(2 l+1) s_{l} h_{l}^{(1)}\left(k_{0} r\right) P_{l}(\cos \theta),
$$

where $s_{l}$ are complex numbers accounting for the scattering coefficients and $h_{l}^{(1)}$ are the spherical Hankel functions of order $l$ and the first kind.

Inside the object, that is, for $r \leq a_{1}$, we have

$$
T^{1}=\sum_{l=0}^{\infty} i^{l}(2 l+1) a_{l} j_{l}\left(k_{1} r\right) P_{l}(\cos \theta),
$$

and finally, inside the shell, that is, for $r \in\left[a_{1}, a_{2}\right]$

$$
T^{2}=\sum_{l=0}^{\infty} i^{l}(2 l+1)\left\{b_{l} j_{l}\left(k_{2} r\right)+c_{l} y_{l}\left(k_{2} r\right)\right\} P_{l}(\cos \theta),
$$

where $a_{l}, b_{l}$, and $c_{l}$ are unknown coefficients that will be determined along with $s_{l}$ by applying the boundary conditions at $r=a_{1}$ and $r=a_{2}$. The boundary conditions involve continuity of the temperature field $T$, as well as its flux $\kappa \nabla T$, or more precisely its radial component $\kappa_{r} \partial_{r} T$.

By denoting the scattering coefficients in a more convenient form, that is,

$$
s_{l}=\frac{-\varsigma_{l}}{\varsigma_{l}+i \beta_{l}}
$$

one finds that

$$
\varsigma_{l}=\operatorname{det}\left(\begin{array}{cccc}
-j_{l}\left(k_{1} a_{1}\right) & y_{l}\left(k_{2} a_{1}\right) & j_{l}\left(k_{2} a_{1}\right) & 0 \\
0 & y_{l}\left(k_{2} a_{2}\right) & j_{l}\left(k_{2} a_{2}\right) & y_{l}\left(k_{0} a_{2}\right) \\
-\kappa_{1} k_{1} j^{\prime}{ }_{l}\left(k_{1} a_{1}\right) & \kappa_{2} k_{2} y^{\prime}{ }_{l}\left(k_{2} a_{1}\right) & \kappa_{2} k_{2} j^{\prime}{ }_{l}\left(k_{2} a_{1}\right) & 0 \\
0 & \kappa_{2} k_{2} y^{\prime}{ }_{l}\left(k_{2} a_{2}\right) & \kappa_{2} k_{2} j^{\prime}{ }_{l}\left(k_{2} a_{2}\right) & \kappa_{0} k j^{\prime}{ }_{l}\left(k_{0} a_{2}\right)
\end{array}\right) \text {, }
$$

and

$$
\beta_{l}=\operatorname{det}\left(\begin{array}{cccc}
-j_{l}\left(k_{1} a_{1}\right) & y_{l}\left(k_{2} a_{1}\right) & j_{l}\left(k_{2} a_{1}\right) & 0 \\
0 & y_{l}\left(k_{2} a_{2}\right) & j_{l}\left(k_{2} a_{2}\right) & j_{l}\left(k_{0} a_{2}\right) \\
-\kappa_{1} k_{1} j^{\prime}{ }_{l}\left(k_{1} a_{1}\right) & \kappa_{2} k_{2} y^{\prime}{ }_{l}\left(k_{2} a_{1}\right) & \kappa_{2} k_{2} j^{\prime}{ }_{l}\left(k_{2} a_{1}\right) & 0 \\
0 & \kappa_{2} k_{2} y^{\prime}{ }_{l}\left(k_{2} a_{2}\right) & \kappa_{2} k_{2} j^{\prime}{ }_{l}\left(k_{2} a_{2}\right) & \kappa_{0} k y^{\prime}{ }_{l}\left(k_{0} a_{2}\right)
\end{array}\right) \text {. }
$$


In these determinants, the wave numbers $k_{j}(j=0,1,2)$, are given by the dispersion relation

$$
k_{j}=\omega \sqrt{\frac{\omega \tau_{j}+i}{\omega \kappa_{j}-i \omega^{2} \tau_{j} \sigma_{j}}},
$$

where one ignores the extra absorption term corresponding to diffuse photon density waves.

\section{B. Ideal cloaking conditions}

Now, we are in a position to compute both analytically and numerically the scattering cross section (SCS) of the system $\Sigma^{\mathrm{sc}}$, which represents the quantity that can be measured by a radar device, placed far away from the structure. In a sense, this scalar physical parameter quantifies how visible (or equivalently invisible) an object or a collection of objects may be to an external observer. In order to render an object completely opaque, one must consider instead the extinction cross section that contains both scattering and absorption, but this goes beyond the scope of this study. The SCS is given by

$$
\Sigma^{\mathrm{sc}}=\frac{4 \pi}{\left|k_{0}\right|^{2}} \sum_{l=0}^{\infty}(2 l+1) \frac{\left|\varsigma_{l}\right|^{2}}{\left|\varsigma_{l}+i \beta_{l}\right|^{2}} .
$$

To make an object invisible or transparent, it is straightforward to see that one needs to have $\Sigma^{\text {sc }}=0$. But this is an impossible task since the SCS involves an infinite number of terms.

A more realistic task is to cancel the first few leading order terms present in Eq. (7). This does not render an object invisible, but in the quasistatic limit, this substantially reduces scattering and is a viable way to realize near perfect invisibility, since only the first (dominant) terms contribute non-negligibly to the scattering.

When the dimension of the system is smaller than the wavelength, $k a_{2} \ll 1$, cancelling the first two terms is sufficient, as $\Sigma^{\text {sc }} \approx 4 \pi /\left|k_{0}\right|^{2}\left(\left|s_{0}\right|^{2}+3\left|s_{1}\right|^{2}\right)$. This leads to

$$
\frac{\tau_{2}-\tau_{0}}{\tau_{2}-\tau_{1}}-\gamma^{3}=0
$$

with the ratio $\gamma=a_{1} / a_{2}$ for $\varsigma_{0}=0$, and

$$
\begin{aligned}
& \frac{\left[\left(\kappa_{0}-\kappa_{2}\right)-i \omega\left(\tau_{0} \sigma_{0}-\tau_{2} \sigma_{2}\right)\right]\left[\left(\kappa_{1}+2 \kappa_{2}\right)-i \omega\left(\tau_{1} \sigma_{1}+2 \tau_{2} \sigma_{2}\right)\right]}{\left[\left(\kappa_{1}-\kappa_{2}\right)-i \omega\left(\tau_{1} \sigma_{1}-\tau_{2} \sigma_{2}\right)\right]\left[\left(\kappa_{0}+2 \kappa_{2}\right)-i \omega\left(\tau_{0} \sigma_{0}+2 \tau_{2} \sigma_{2}\right)\right]} \\
& \quad-\gamma^{3}=0,
\end{aligned}
$$

for $\varsigma_{1}=0$.

Figure 2(a) plots the solution of Eq. (15) for varying ratio $\gamma$ and thermal relaxation time $\tau_{1}$ (in units of $\tau_{0}$ ). One can observe a classical behavior in its lower part for $k_{0} a_{1}=0.5$. The white region corresponds to negative values of $\tau_{2}$, and is henceforth ignored since it is unphysical, whereas the red part corresponds to values of $\tau_{2}$ in the range of $0.1 \tau_{0}$ to $3.5 \tau_{0}$. The upper black curve stands for $\tau_{2}=0$ (i.e., $\gamma^{3} \tau_{1} / \tau_{0}=1$ ). Equation (16) is, however, different from other scenarios (heat waves of DPDWs) as its first term is complex valued. To realize perfect dipole scattering cancellation, one has to cancel both its real and imaginary parts. However, by decomposing it, one can observe that the imaginary part takes close-to-zero values, except for $\kappa_{2}=\kappa_{1}$ and $\kappa_{2}=-1 / 2 \kappa_{0}$. Thus, it is possible to cancel the real part of Eq. (16) and exclude these values $\left(\kappa_{1}\right.$ and $-1 / 2 \kappa_{0}$ ) from the possible solutions. The real part of the left-hand side of Eq. (16) can be cast in the form of

$$
A / B-\gamma^{3}=0
$$

with,

$$
A=\left(x_{1}-x_{2}\right)\left(x_{3}+x_{4}\right)\left(x_{5}-x_{6}\right)\left(x_{7}+x_{8}\right),
$$

and

$$
B=\left(x_{3}^{2}+x_{4}^{2}\right)\left(x_{7}^{2}+x_{8}^{2}\right),
$$

where $x_{1}=\kappa_{0}-\kappa_{2}, x_{2}=\omega\left(\tau_{0} \sigma_{0}-\tau_{2} \sigma_{2}\right), x_{3}=\kappa_{1}-\kappa_{2}$, $x_{4}=\omega\left(\tau_{1} \sigma_{1}-\tau_{2} \sigma_{2}\right), x_{5}=\kappa_{1}+2 \kappa_{2}, x_{6}=\omega\left(\tau_{1} \sigma_{1}+2 \tau_{2} \sigma_{2}\right)$, $x_{7}=\kappa_{0}+2 \kappa_{2}$, and $x_{8}=\omega\left(\tau_{0} \sigma_{0}+2 \tau_{2} \sigma_{2}\right)$.

Equation (17) is of fourth-order, so one expects to have four possible solutions for $\kappa_{2}$. This is exactly what one can observe from Figs. 2(b) and 2(c), where four branches can be distinguished from the three-dimensional plot. Figure 2(c) plots the solutions (contour plots) for different values of the ratio $\gamma$, that is, $0.01,0.5$, and 0.95 , from left to right, respectively. For each value of $\kappa_{1}$ (and $\gamma$ ), one has four possible solutions for $\kappa_{2}$ that cancel Eq. (17).

\section{Scattering cancellation technique}

The considered geometry is shown in Fig. 1(a) with radius $a_{1}=1 \mathrm{~cm}$, and we choose the radius of the shell $a_{1}=1.15 \mathrm{~cm}$ (or $\gamma=0.87$ ), which corresponds to a relatively thin cloaking shell. The parameters of the object are $\kappa_{1}=3 \kappa_{0}$ and $\tau_{1} \sigma_{1}=\tau_{0} \sigma_{0}$. Then, the SCS is numerically computed for a finite scattering order $N_{0}$. In fact, it has been shown in many recent studies that the scattering coefficients $\left|s_{l}\right|$ are of the order $\left|k_{0} a_{1}\right|^{2 l+1}$, so when $\left|k_{0} a_{1}\right| \ll 1$, only a few coefficients are enough to reach convergence of $\Sigma^{\text {sc }}$. In this study, one verifies convergence, and chooses $N_{0}=10$. The SCS of the structure is thus normalized with the SCS of the bare object and plotted in logarithmic scale (to base 10) in Fig. 3(a) vs the diffusivity coefficient $\kappa_{2}$ (in units of $\kappa_{0}$ ) and the thermal relaxation time $\tau_{2}$ (in units of $\left.\tau_{0}\right)$. It can be seen from this two-dimensional (2D) plot that the normalized SCS is increased in some regions (dark red color), which corresponds to enhanced scattering as well as reduced scattering (dark blue color), which corresponds 
(a)

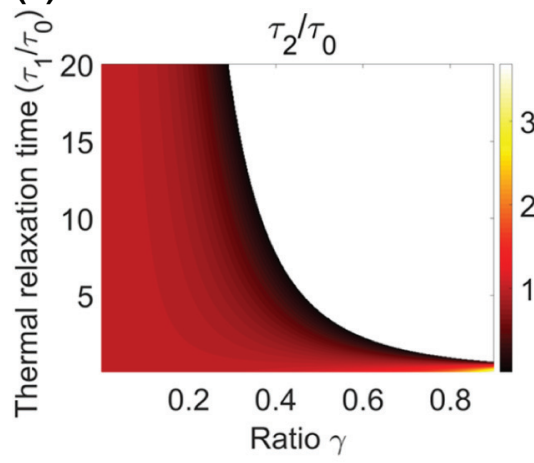

(b)

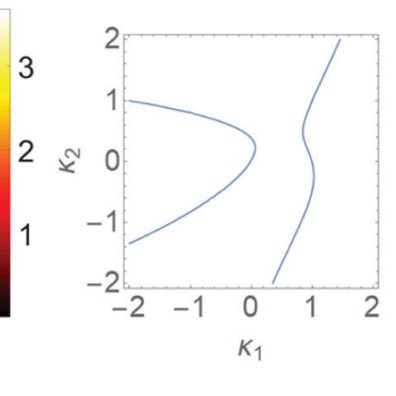

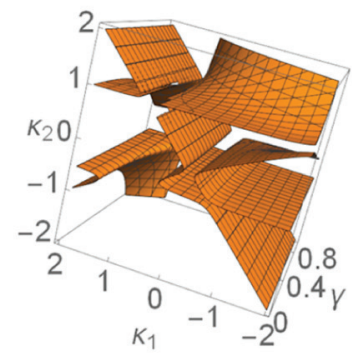

FIG. 2. (a) Monopole scattering cancellation deduced from Eq. (15). (b) Dipole scattering cancellation for the imaginary and real parts deduced from Eq. (16). (c) Cross-section plots for $\gamma=0.01,0.5$ and 0.95 , respectively (from left to right).

(c)
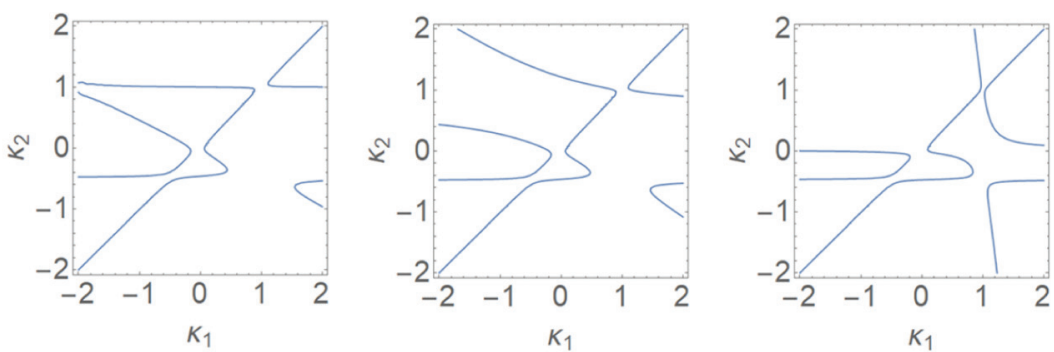

to transparency (or cloaking) effect. In particular, a minimum of $\Sigma_{2}^{\mathrm{sc}}$ is obtained around the values $\kappa_{2}=0.6 \kappa_{0}$ and $\tau_{2}=0.3 \tau_{0}$ [dark spot in Fig. 3(a)].

To isolate the effect of $\kappa_{2}$ and $\tau_{2}$ on the scattering reduction, a plot of $\Sigma_{2}^{\text {sc }}$ is given vs $\kappa_{2}$ for different values of $\tau_{2}$ in Fig. 3(b). One can see that two regimes exist. First, for small values of $\tau_{2}$, no reduction is significantly obtained for all values of $\kappa_{2}$ [red line on the bottom of Fig. 3(a)]. Then the scattering reduction regime starts operating and is maximal for $\tau_{2}=0.3 \tau_{0}$, and again starts (a)

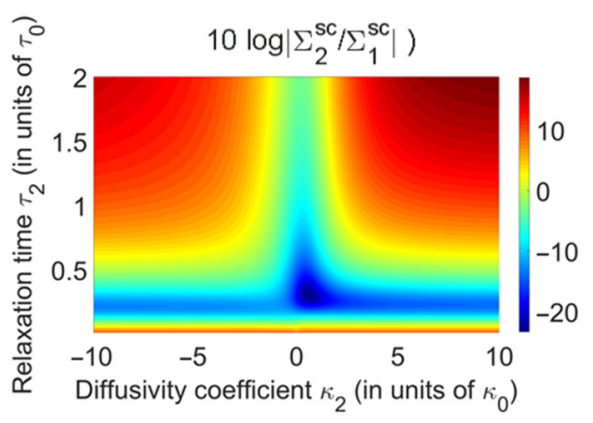

(c)

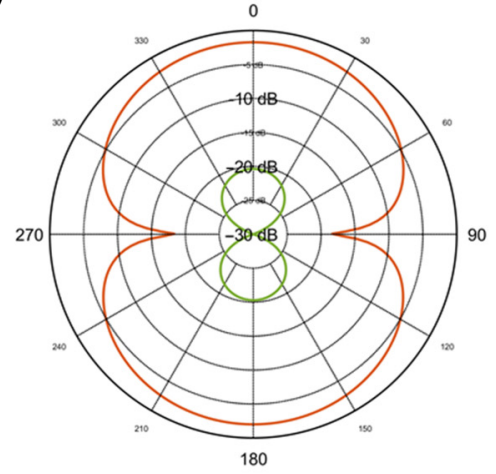

(b)

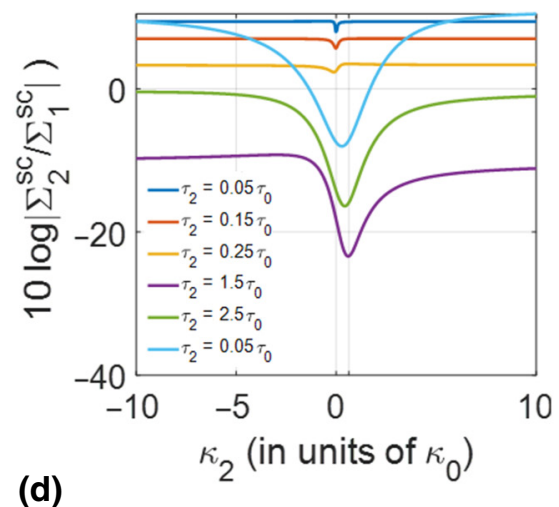

(d)

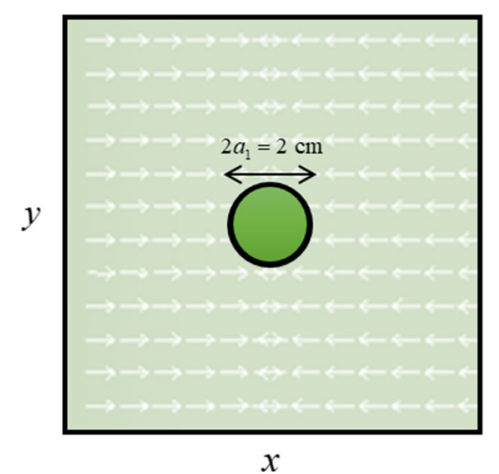

FIG. 3. (a) Scattering cross section in logarithmic scale (to base 10) vs the normalized diffusivity coefficient $\kappa_{2}$ and thermal relaxation time $\tau_{2}$. (b) Crosssection plots from the 2D graph [in Fig. 3(a)] for different values of $\tau_{2}$. (c) Far-field polar scattering amplitude for both the uncloaked object (red line) and cloaked one (green line). Near-field plot of the temperature field in the vicinity of the core-shell structure. 


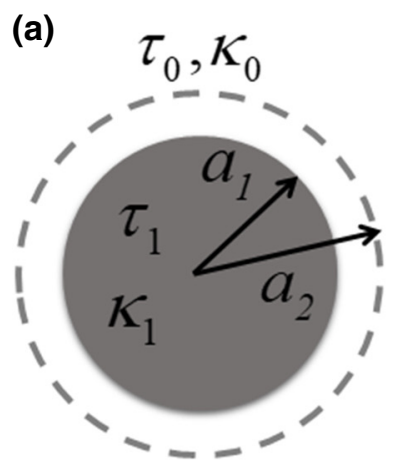

(b)

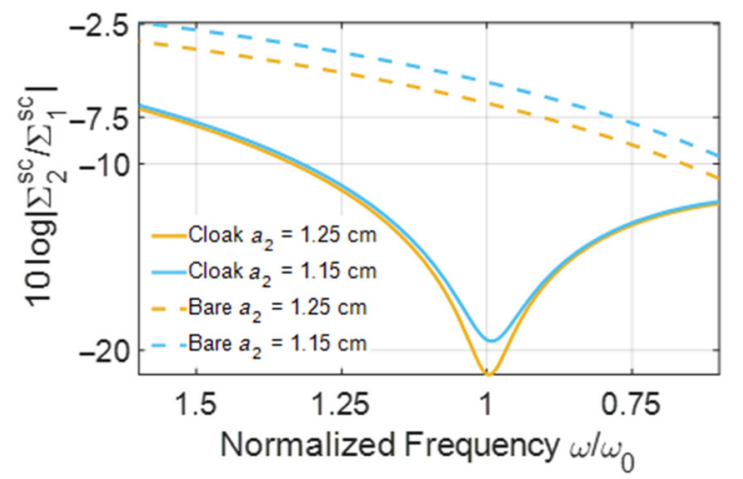

FIG. 4. (a) Scheme of the object surrounded by the metasurface cloak of radius $a_{2}$. (b) SCS in logarithmic scale (to base 10) vs the normalized frequency $\omega / \omega_{0}$ for the object and cloaked object for two different radii of the metasurface. gradually disappearing. This shows that effective cloaking can be obtained only by optimizing both parameters $\left(\tau_{2}\right.$ and $\kappa_{2}$ ) of the shell, unlike in static studies, where only $\kappa_{2}$ is considered. One goes one step further by analyzing the SCS in polar coordinates, by imposing the values of $\kappa_{2}$ and $\tau_{2}$ that better reduce the SCS, and plotting $\Sigma_{2}^{\text {sc }}$ in the farfield vs the angle of observation $\theta$. Figure 3(c) shows such a plot, and a significant reduction of $\Sigma_{2}^{\mathrm{sc}}$ is obtained for all angles by using the cloaking shell. The near-field plot of the temperature field is further given in Fig. 3(d) in the presence of the same core-shell structure for $k_{0} a_{1}=0.5$, and shows no perturbation of the amplitude of the field in the vicinity of the cloaked object, thus demonstrating the robustness of the SCS for such kind of waves.

\section{MANTLE CLOAKING}

Mantle cloaking has been shown to make possible considerable scattering reduction for several kinds of waves $[7,25,26,31]$ using an ultrathin metasurface cloak. It relies on coating an object with a metasurface (ideally 2D) with tailored impedance to cancel the radar scattering. In the case of Maxwell-Cattaneo heat waves, the boundary conditions at the interface $r=a_{1}$ is the same as in Section C (i.e., continuity of $T$ and its flux $\kappa_{r} \partial_{r} T$ ). However, at the boundary $r=a_{2}$ (i.e., at the metasurface), one has $T\left(r=a_{2}^{-}\right)=T\left(r=a_{2}^{+}\right)$, where the - and + signs stand for the inside and outside of the metasurface, respectively, as shown in Fig. 4(a). The second condition is

$$
\begin{aligned}
& \left(\kappa_{0}-i \omega \sigma_{0} \tau_{0}\right) \frac{\partial T}{\partial r}\left(r=a_{2}^{+}\right)-\left(\kappa_{2}-i \omega \sigma_{2} \tau_{2}\right) \frac{\partial T}{\partial r}\left(r=a_{2}^{-}\right) \\
& =Z_{\mathrm{MC}}^{-1} T\left(a_{2}\right)
\end{aligned}
$$

with $Z_{\mathrm{MC}}$ the average surface impedance of MaxwellCattaneo heat waves relating the temperature $T$ to its flux $\kappa_{r} \partial_{r} T$. The scattering coefficients in this configuration become

$$
\varsigma_{l}=\operatorname{det}\left(\begin{array}{cccc}
-j_{l}\left(k_{1} a_{1}\right) & y_{l}\left(k_{0} a_{1}\right) & j_{l}\left(k_{0} a_{1}\right) & 0 \\
0 & y_{l}\left(k_{0} a_{2}\right) & j_{l}\left(k_{0} a_{2}\right) & y_{l}\left(k_{0} a_{2}\right) \\
-\kappa_{1} k_{1} j^{\prime}{ }_{l}\left(k_{1} a_{1}\right) & \kappa_{0} k_{2} y^{\prime}{ }_{l}\left(k_{0} a_{1}\right) & \kappa_{0} k_{2} j^{\prime}{ }_{l}\left(k_{0} a_{1}\right) & 0 \\
0 & y^{\prime}{ }_{l}\left(k_{0} a_{2}\right)+\chi y_{l}\left(k_{0} a_{2}\right) & j^{\prime}{ }_{l}\left(k_{0} a_{2}\right)+\chi j_{l}\left(k_{0} a_{2}\right) & j^{\prime}{ }_{l}\left(k_{0} a_{2}\right)
\end{array}\right) \text {, }
$$

with $\chi=i \omega /\left[Z_{\mathrm{MC}} k_{0}\left(\kappa_{0}-i \omega \sigma_{0} \tau_{0}\right)\right]$ a dimensionless function. For $l=0$, and by imposing $\varsigma_{0}=0$, we get

$X_{\mathrm{MC}}=\frac{2}{3 \gamma^{3} \omega a_{1}}\left(\kappa_{0} \gamma^{3}+\operatorname{Re}\left[\frac{\left(\kappa_{0}-i \omega \sigma_{0} \tau_{0}\right)+2\left(\kappa_{1}-i \omega \sigma_{1} \tau_{1}\right)}{\left(\kappa_{1}-i \omega \sigma_{1} \tau_{1}\right)-\left(\kappa_{0}-i \omega \sigma_{0} \tau_{0}\right)}\right]\right)$,

by denoting $X_{\mathrm{MC}}=\operatorname{Im}\left[Z_{\mathrm{MC}}\right]$ the reactive part of the total impedance.

Figure 4(b) gives the SCS of the structure shown in Fig. 4(a), that is, the object surrounded by the mantle cloak with $a_{1}=1 \mathrm{~cm}$ and $a_{2}=1.15 \mathrm{~cm}$. It can be clearly seen that a substantial scattering reduction can be achieved around the design wave number $k_{0} a_{1}=0.5$ (that corresponds to $\left.\omega_{0}\right)$.

\section{CONCLUSIONS}

In summary, this study presents the first demonstration of scattering cancellation cloaking for heat waves obeying the Maxwell-Cattaneo transfer law, which permits to avoid unphysical features associated with Fourier transfer law, 
such as instantaneous diffusion. This problem can become a key issue in the analysis of time-modulated media since the Fourier heat equation is not frame invariant. In a moving medium it reads as $\partial_{t} T+\boldsymbol{u} \cdot \nabla T-\kappa \Delta T=\delta$ where $\boldsymbol{u}$ is the velocity taken at a given point of the moving medium. When the velocity vanishes, one retrieves the usual Fourier equation. Since this frame-invariant equation is itself parabolic, it suffers from the same pitfall as the Fourier equation, any change in temperature at a given point of the moving medium is felt instantaneously elsewhere. A frame-invariant-form of the Maxwell-Catteneo equation solves this issue [43] and should thus be preferred in the analysis of heat problems in time-modulated media. The time relaxation parameter becomes all the more important because the time-modulation is fast.

Both SCT and mantle cloaking are analyzed for this kind of heat waves and are shown to result in near-perfect invisibility in all directions. The far-field and near-field numerical calculations demonstrate the robustness of such thin cloaks, which should be fairly easy to fabricate, due to the isotropy and homogeneity of the cloaking shell parameters (thermal conductivity and thermal relaxation time), unlike TO-based cloaks. Here, we wish to add a comment about controlling the thermal relaxation time. For instance, the relaxation time can be tuned by generating some time modulations in a way similar to that proposed in Ref. [44]. The faster the time modulation, the larger the relaxation time. Note that a Maxwell-Cattaneo equation would be a good way to analyze such nonreciprocal media.

\section{ACKNOWLEDGMENT}

S.G. wishes to thank a visiting position in the group of Professor R.V. Craster at Imperial College London funded by EPSRC program "Mathematical Fundamentals of Metamaterials for Multiscale Physics and Mechanics" (Grant No. EP/L024926/1).

[1] A. Alu and N. Engheta, Achieving transparency with plasmonic and metamaterial coatings, Phys. Rev. E 72, 016623 (2005).

[2] D. Rainwater, A. Kerkhoff, K. Melin, J. C. Soric, G. Moreno, and A. Alu, Experimental verification of threedimensional plasmonic cloaking in free-space, New J. Phys. 14, 013054 (2012).

[3] F. Bilotti, S. Tricarico, and L. Vegni, Plasmonic metamaterial cloaking at optical frequencies, IEEE Trans. Nanotechnol. 9, 55 (2010).

[4] M. D. Guild, A. Alu, and M. R. Haberman, Cancellation of acoustic scattering from an elastic sphere, J. Acoust. Soc. Am. 129, 1355 (2011).

[5] M. Farhat, P.-Y. Chen, H. Bagci, S. Guenneau, S. Enoch, and A. Alu, Platonic scattering cancellation for bending waves in a thin plate, Sci. Rep. 4, 4644 (2014).
[6] P. Y. Chen, M. Farhat, S. Guenneau, S. Enoch, and A. Alu, Acoustic scattering cancellation via ultrathin pseudosurface, Appl. Phys. Lett. 99, 191913 (2011).

[7] M. Farhat, P. Y. Chen, S. Guenneau, S. Enoch, and A. Alu, Frequency-selective surface acoustic invisibility for threedimensional immersed objects, Phys. Rev. B 86, 174303 (2012).

[8] M. D. Guild, M. R. Haberman, and A. Alu, Plasmonic cloaking and scattering cancelation for electromagnetic and acoustic waves, Wave Motion 48, 468 (2011).

[9] M. Farhat, P.-Y. Chen, H. Bagci, C. Amra, S. Guenneau, and A. Alu, Thermal invisibility based on scattering cancellation and mantle cloaking, Sci. Rep. 5, 9876 (2015).

[10] R. Fleury and A. Alu, Quantum cloaking based on scattering cancellation, Phys. Rev. B 87, 045423 (2013).

[11] J. B. Pendry, D. Schurig, and D. R. Smith, Controlling electromagnetic fields, Science 312, 1780 (2006).

[12] D. Schurig, J. J. Mock, B. J. Justice, S. A. Cummer, J. B. Pendry, A. F. Starr, and D. R. Smith, Metamaterial electromagnetic cloak at microwave frequencies, Science 314, 977 (2006).

[13] U. Leonhardt, Optical conformal mapping, Science 312, 1777 (2006).

[14] G. W. Milton and N. A. P. Nicorovici, On the cloaking effects associated with anomalous localized resonance, Proc. R. Soc. A 462, 3027 (2006).

[15] N. A. P. Nicorovici, G. W. Milton, R. C. McPhedran, and L. C. Botten, Quasistatic cloaking of two-dimensional polarizable discrete systems by anomalous resonance, Opt. Express 15, 6314 (2007).

[16] W. S. Cai, U. K. Chettiar, A. V. Kildishev, and V. M. Shalaev, Optical cloaking with metamaterials, Nat. Photonics 1, 224 (2007).

[17] M. Farhat, S. Guenneau, S. Enoch, A. B. Movchan, F. Zolla, and A. Nicolet, A homogenization route towards square cylindrical acoustic cloaks, New J. Phys. 10, 115030 (2008).

[18] I. I. Smolyaninov, V. N. Smolyaninova, A. V. Kildishev, and V. M. Shalaev, Anisotropic Metamaterials Emulated by Tapered Waveguides: Application to Optical Cloaking, Phys. Rev. Lett. 102, 213901 (2009).

[19] Y. Lai, H. Chen, Z.-Q. Zhang, and C. Chan, Complementary Media Invisibility Cloak that Cloaks Objects at a Distance Outside the Cloaking Shell, Phys. Rev. Lett. 102, 093901 (2009).

[20] M. Maldovan, Sound and heat revolutions in phononics, Nature 503, 209 (2013).

[21] S. Guenneau and C. Amra, Anisotropic conductivity rotates heat fluxes in transient regimes, Opt. Express 21, 6578 (2013).

[22] S. Guenneau, C. Amra, and D. Veynante, Transformation thermodynamics: cloaking and concentrating heat flux, Opt. Express 20, 8207 (2012).

[23] T. C. Han, X. Bai, D. L. Gao, J. T. L. Thong, B. Li, and C.W. Qiu, Experimental Demonstration of a Bilayer Thermal Cloak, Phys. Rev. Lett. 112, 054302 (2014).

[24] R. Schittny, M. Kadic, S. Guenneau, and M. Wegener, Experiments on Transformation Thermodynamics: Molding the Flow of Heat, Phys. Rev. Lett. 110, 195901 (2013). 
[25] H. Y. Xu, X. H. Shi, F. Gao, H. D. Sun, and B. L. Zhang, Ultrathin Three-Dimensional Thermal Cloak, Phys. Rev. Lett. 112, 054301 (2014).

[26] M. Farhat, P.-Y. Chen, S. Guenneau, H. Bagci, K. N. Salama, and A. Alu, Cloaking through cancellation of diffusive wave scattering, Proc. R. Soc. A 472, 20160276 (2016).

[27] M. Farhat, S. Guenneau, T. Puvirajesinghe, and F. H. Alharbi, Frequency domain transformation optics for diffusive photon density waves' cloaking, Opt. Express 26, 24792 (2018).

[28] R. V. Craster, S. R. L. Guenneau, H. R. Hutridurga, and G. A. Pavliotis, Cloaking via mapping for the heat equation, Multiscale Model. Simul. 16, 1146 (2018).

[29] H. J. Li and H. Y. Liu, On anomalous localized resonance and plasmonic cloaking beyond the quasi-static limit, Proc. R. Soc. A 474, 20180165 (2018).

[30] A. Alu and N. Engheta, Plasmonic materials in transparency and cloaking problems: mechanism, robustness, and physical insights, Opt. Express 15, 3318 (2007).

[31] A. Alu, Mantle cloak: Invisibility induced by a surface, Phys. Rev. B 80, 245115 (2009).

[32] P. Y. Chen and A. Alu, Mantle cloaking using thin patterned metasurfaces, Phys. Rev. B 84, 205110 (2011).

[33] A. Monti, A. Toscano, and F. Bilotti, in Proc. IEEE Antennas Propag. Soc. Int. Symp. (APSURSI) (2012), pp. 1-2.

[34] Y. R. Padooru, A. B. Yakovlev, P. Y. Chen, and A. Alu, Analytical modeling of conformal mantle cloaks for cylindrical objects using sub-wavelength printed and slotted arrays, J. Appl. Phys. 112, 034907 (2012).
[35] C. I. Christov, On frame indifferent formulation of the Maxwell-Cattaneo model of finite-speed heat conduction, Mech. Res. Commun. 36, 481 (2009).

[36] M. Ostoja-Starzewski, A derivation of the MaxwellCattaneo equation from the free energy and dissipation potentials, Int. J. Eng. Sci. 47, 807 (2009).

[37] D. D. Joseph and L. Preziosi, Heat waves, Rev. Mod. Phys. 61, 41 (1989).

[38] A. Einstein, A brief outline of the development of the theory of relativity, Nature 106, 782 (1921).

[39] T. Hayat, M. Javed, M. Imtiaz, and A. Alsaedi, Effect of Cattaneo-Christov heat flux on Jeffrey fluid flow with variable thermal conductivity, Results Phys. 8, 341 (2018).

[40] F. Ekoue, D. Gigon, G. Plantamp, and E. Zajdman, Maxwell-Cattaneo regularization of heat equation, Int. J. Math. Comput. Phys. Electr. Comput. Eng. 7, 772 (2013).

[41] M. Zhang, A relationship between the periodic and the Dirichlet BVPs of singular differential equations, Proc. R. Soc. A 128, 1099 (1998).

[42] D. Zhang and M. Ostoja-Starzewski, Telegraph equation: two types of harmonic waves, a discontinuity wave, and a spectral finite element, Acta Mech. 230, 1725 (2019).

[43] C. Christov and P. Jordan, Heat Conduction Paradox Involving Second-Sound Propagation in Moving Media, Phys. Rev. Lett. 94, 154301 (2005).

[44] D. Torrent, O. Poncelet, and J.-C. Batsale, Nonreciprocal Thermal Material by Spatiotemporal Modulation, Phys. Rev. Lett. 120, 125501 (2018). 